TRANSACTIONS OF THE

AMERICAN MATHEMATICAL SOCIETY

Volume 335, Number 2, February 1993

\title{
PIECEWISE $\mathrm{SL}_{2} \mathrm{Z}$ GEOMETRY
}

\author{
PETER GREENBERG
}

\begin{abstract}
Piecewise $\mathrm{SL}_{2} \mathrm{Z}$ geometry studies properties of the plane invariant under pl-homeomorphisms which, locally, have the form $x \mapsto A x+b$, with $A \in \mathrm{SL}_{2} \mathbf{Z}, b \in \mathbf{Q}^{2}$, and whose singular lines are rational. In this paper, invariants of polygons are obtained, relations with Pick's theorem are described, and a conjecture is posed.
\end{abstract}

\section{INTRODUCTION}

The classic Pick's theorem (see [GKW]) asserts that if $P$ is a polygon whose vertices have integral coordinates (an integral polygon) then the number of points of $\mathbf{Z}^{2}$ in the interior of $P$ is area $(P)-\frac{1}{2} \#\left(\partial P \cap \mathbf{Z}^{2}\right)+1$ (here \# denotes cardinality). Looking behind the proof, we are led to consider a certain graph $G_{1} P$ and associated simplicial complex $K_{1} P$ associated to $P$. The complex $K_{1} P$ can be thought of as the space of triangulations of $P$; it turns out (1.13) that if area $(P)>1$, then $K_{1} P$ is a pl-disk.

One motivation for this study is to understand the geometry of integral polygons and the piecewise $\mathrm{SL}_{2} \mathrm{Z}$ maps between them, that is, piecewise linear maps which, in each "piece", have the form

$$
f(x, y)=A(x, y)+v, \quad A \in \mathrm{SL}_{2} \mathbf{Z}, v \in \mathbf{Q}^{2} .
$$

The classifying space of the pseudogroup $\Gamma$ of such homeomorphisms is rather simple-roughly [Gr] a CW complex with a finite number of cells in each dimension-and it would be interesting to see this reflected in the geometry. We calculated in $[\mathrm{Gr}]$ that, in a homological sense, the only quantities of closed integral polygons invariant under $\Gamma$ are the area and a sort of "length" (1.2). Here we prove this in a stronger, geometric sense (1.3).

The group $G$ of germs at $(0,0)$ of the pseudogroup $\Gamma$ contains a group $F^{\prime}$ which is an "algebraic delooping" of the braid group [GS]. Thinking of $\Gamma$ as a globalization of $G$, it makes sense to look for connections with the braid groups. As was noted by Devaney in [D], if we restrict the $v$ in (*) to lie in $\mathbf{Z}^{2}$, then piecewise $\mathrm{SL}_{2} \mathbf{Z}$ maps permute the points $\frac{1}{N} \mathbf{Z}^{2}$ for each $N$. Thus, if $\operatorname{Aut}_{1}(P, \partial)$ denotes the group of such automorphisms of $P$, fixing the boundary, there are evident homomorphisms from $\operatorname{Aut}_{1}(P, \partial)$ to certain braid groups. (See Figure 1.)

Received by the editors October 9, 1990.

1991 Mathematics Subject Classification. Primary 52A25.

Key words and phrases. Piecewise linear homeomorphisms. 

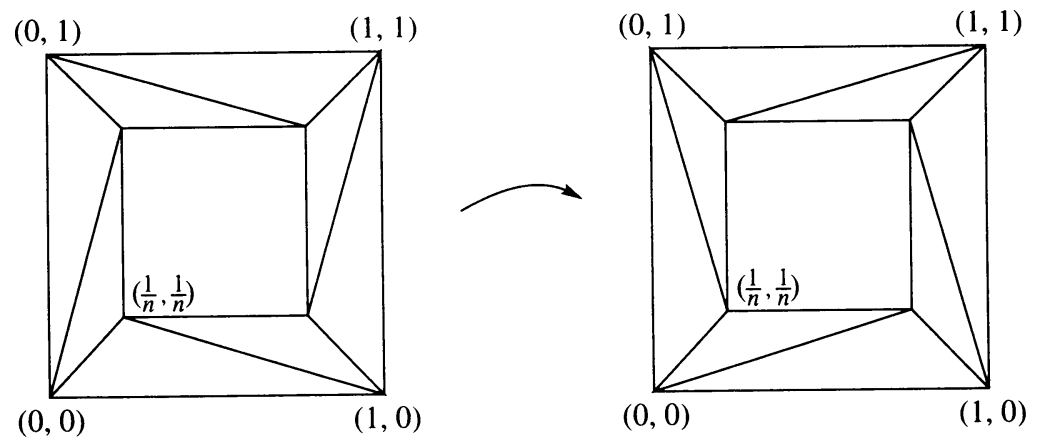

Figure 1. A “ $\frac{1}{4}$ Dehn twist” in $\operatorname{Aut}_{1}(P, \partial)$

However, perhaps one should look for deeper structural relations between $\operatorname{Aut}_{1}(P, \partial)$ and braid or mapping class groups. By taking a limit of complexes $K_{1} N P$, one arrives at a space $K(P)$ on which $\operatorname{Aut}_{1}(P, \partial)$ acts (1.16). The space $K(P)$ is analogous to the "complexes of curves" which arise in connection with the mapping class groups.

Conversations with Javier Bracho, Stephen Halperin, and Victor Neumann have been helpful during this work. The hospitality of the Geometry Group at the Université de Lille is gratefully acknowledged.

\section{Definitions AND MAIN RESUltS}

We begin by defining certain pseudogroups of pl-homeomorphisms between open subsets of $\mathbf{R}^{2}$. We will denote by $\frac{1}{N} \mathbf{Z}$ the subgroup of $\mathbf{Q}$ generated by $\frac{1}{N}$, and by $A_{N}$ (resp. $A_{0}$ ) the affine extension of $\frac{1}{N} \mathbf{Z}^{2}$ (resp. $\mathbf{Q}^{2}$ ) generated by $\mathrm{SL}_{2} \mathbf{Z}$. A rational line (resp. integral line) is a line passing through two rational (resp. integral) points in the plane.

1.1. Definition. A $p \mathbf{Z}_{N}$ homeomorphism is an orientation-preserving homeomorphism $g: U \rightarrow V$ between open subsets of the plane, such that there exists a finite set of rational lines $\left\{l_{i}\right\}$ such that $g$ agrees with some element $g_{C}$ of $A_{N}$ on any component $C$ of $U-\amalg l_{i}$. A $p \mathbf{Z}$ homeomorphism is a $p Z_{1}$ homeomorphism, in which we require the lines $l_{i}$ to be integral.

Before discussing invariants, we establish some notation for polygonal curves.

In this paper, a polygonal curve means a curve made of a finite number of rational line segments between rational points of $\mathbf{R}^{2}$; the endpoints of the segments of an integral polygonal curve are required to lie in $\mathbf{Z}^{2}$. If $v_{i} \in \mathbf{Q}^{2}$, we denote by $\overline{v_{0} \cdots v_{n}}$ the polygonal curve made of segments $\bar{v}_{k} v_{k+1}$. An (integral) polygon is a simple closed (integral) polygonal curve. We write int $P$ for the open set enclosed by a polygon $P$, and $\overline{\text { int } P}$ for the closure of int $P$. Finally, $\mathscr{C}$ and $P$ denote the sets of polygonal curves and polygons.

If $P$ is a polygon, the area $a(P)$ of int $P$ is invariant under $p Z_{0}$ maps. There is also an invariant "length."

1.2. Proposition. There is a function $L: \mathscr{C} \rightarrow \mathbf{Q}$ which takes positive values, such that

(a) (invariance) If $P \in \mathscr{C}, P \subseteq U$, and $g: U \rightarrow V$ is a p $\mathbf{Z}_{0}$-homeomorphism, then $L(P)=L(g(P))$.

(b) (subdivision) $L\left(\overline{v_{0} \cdots v_{n}}\right)=L\left(\overline{v_{0} \cdots v_{k}}\right)+L\left(\overline{v_{k} \cdots v_{n}}\right)$. 
(c) (homothety) $L(N P)=N L(P), P \in \mathscr{C}$, where $N P$ is the image of $P$ under the map $(x, y) \rightarrow(N x, N y)$.

(d) (no metric) If $a, b \in \mathbf{Q}^{2}$, then $\inf \left(\overline{a v_{1} \cdots v_{n-1} b}\right)=0$.

Proof. If $a=(p / N, q / N), b=(r / N, s / N)$, and $p, q, r, s \in \mathbf{Z}$, we define $L(\overline{a b})=\frac{1}{N}\left(\#\left(\frac{1}{N} \mathbf{Z}^{2} \cap \overline{a b}\right)-1\right)$, where $\# X$ denotes the cardinality of a set $X$. Observe that $L(\overline{a b})$ is independent of the $N$ used. Extend $L$ to all of $\mathscr{C}$ so as to satisfy (b). Property (a) (invariance) is a consequence of the fact that $\mathrm{SL}_{2} \mathrm{Z}$ preserves the lattices $\frac{1}{N} \mathbf{Z}^{2}$, and property (c) is a quick calculation. To prove (d), by (a) and (c) it suffices to take $a=(0,0)$ and $b=(2,0)$. Then note that $L(\overline{a(1,1 / k) b})=2 / k$.

The following theorem says that $a$ and $L$ are the only invariants of the action of $p \mathbf{Z}_{0}$ homeomorphisms on $\mathscr{P}$.

1.3. Theorem. Let $P, Q \in \mathscr{P}$, with $a(P)=a(Q)$ and $L(P)=L(Q)$. Let $p \in P$ and $q \in Q$ be rational points. Then there exists a $p \mathbf{Z}_{0}$ homeomorphism $g: \overline{\text { int } P} \rightarrow \overline{\operatorname{int} Q}$ such that $g(p)=q$.

If $P, Q$ are integral polygons, and $p, q \in \mathbf{Z}^{2}$, we may choose $g$ to be a $p \mathrm{Z}$ homeomorphism.

The proof of the theorem is somewhat involved, so we postpone it to $\S 2$. The main idea, that of a triangulation, will now be applied to reproduce the proof [GKW] of a theorem of Pick.

1.4. Proposition (Pick). Let $P$ be an integral polygon. The number of points of $\mathbf{Z}^{2}$ in int $P$ is

$$
a(P)-\frac{1}{2} \#\left(P \cap \mathbf{Z}^{2}\right)+1 .
$$

(Note that $\left.\#\left(P \cap \mathbf{Z}^{2}\right)=L(P)\right)$. The proof requires the following notions.

1.5. Definition. An $N$-segment $\overline{a b}$ is a segment so that $\overline{a b} \cap \frac{1}{N} \mathbf{Z}^{2}=\{a, b\}$. An $N$-triangle is a triangle $\overline{a b c a}$ whose sides are $N$-segments, and whose interior contains no points of $\frac{1}{N} \mathbf{Z}^{2}$. If $P=\overline{v_{0} \cdots v_{n} v_{0}}$ is a polygon and $v_{i} \in \frac{1}{N} \mathbf{Z}^{2}$, then an $N$-triangulation of $P$ is a triangulation by $N$-triangles.

1.6. Lemma. The length $L(\overline{a b})$ of an $N$-segment is $\frac{1}{N}$. The area of an $N$ triangle is $\frac{1}{N^{2}} \frac{1}{2}$.

Proof. The first statement follows from the definition. For the second, it suffices to take $N=1$. Further, after transformation by an element of $A_{1}$, we may choose the vertices of the triangle at $(0,0),(1,0)$, and $(a, b)$, where $b>0$, $a, b \in \mathbf{Z}$.

Consider the parallelogram $P$ with corners $(0,0),(1,0),(a, b)$, and $(a-$ $1, b)$. It suffices to show that $a(P)=1$, or that $(1,0),(a-1, b)$ is a basis for $\mathbf{Z}^{2}$. But since int $P \cap \mathbf{Z}^{2}$ is empty, int $P^{\prime} \cap \mathbf{Z}^{2}$ is also empty for any $P^{\prime}$ in the tesselation of $\mathbf{R}^{2}$ by copies of $P$. Hence, $(1,0),(a-1, b)$ is a basis for $\mathbf{Z}^{2}$.

Proof of 1.4. Let $P$ be an integral polygon, and let $V, E$, and $T$ be the numbers of vertices, edges, and triangles in a 1-triangulation. (It will be obvious presently that 1-triangulations exist.) Then $T=2 a(P)$ and the number of edges 


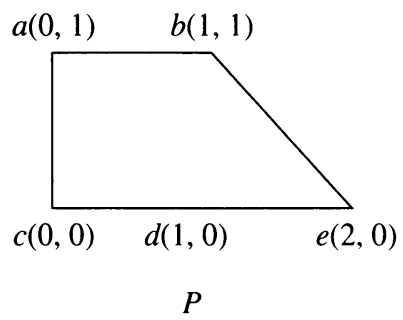

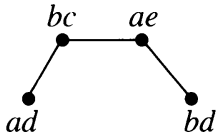

$G(P)$

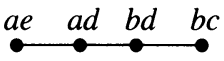

$K(P)$

FIGURE 2

on $P$ is $L(P)=\#\left(P \cap \mathbf{Z}^{2}\right)$. Each triangle has three edges, and the edges not on the boundary share two triangles, so

$$
E=\frac{3 T}{2}+\frac{1}{2} L(P)=3 a(P)+\frac{1}{2} L(P) .
$$

By Euler's formula, $V=1+E-T=a(P)+\frac{1}{2} L(P)+1$. But the number of vertices on $P$ is $L(P)$ so the number of vertices in int $P$ is $a(P)-\frac{1}{2} \#\left(P \cap Z^{2}\right)+1$.

In order to investigate triangulations of polygons, we introduce graphs $G_{N} P$ associated to integral polygons $P$. The vertices of $G_{N} P$ are $N$-segments whose interior is contained in the interior of $P$. If two $N$-segments intersect in their interiors, then there is an edge between two vertices and we say the $N$-segments cross. See Figure 2.

Now, if $G$ is a graph, an independent subset of $G$ (see [G]) is a set of vertices $\left\{v_{i}\right\}$ so that there is no edge between any $v_{i}$ and $v_{j}$. Let $K(G)$ denote the simplicial complex whose $k$-simplices are independent subsets of $G$ of cardinality $k+1$; write $K_{N} P$ for $K\left(G_{N}(P)\right)$. We consider $G_{N} P$ because of the following:

1.8. Remark. A maximal independent set of $G_{N} P$ is precisely the set of $N$ segments, not in $P$, in an $N$-triangulation of $P$.

1.9. Proposition. Let $P$ be an integral polygon. The maximal independent sets of $G_{N} P$ have $3 N^{2} a(P)-\frac{N}{2} L(P)$ members.

Proof. For $N=1$, this is just equation (1.7), with the observation that $P$ contains $L(P)$ edges. For general $N$, apply the homothety $(x, y) \rightarrow(N x, N y)$ to change an $N$-triangulation of $P$ to a 1-triangulation of $N P$.

A graph with the property that all of its maximal independent sets have the same cardinality is called well-covered (see [G]). The $G_{N}(P)$ seem to be new examples of well-covered graphs.

In some sense, the structure of $G_{N} P$ stabilizes as $N$ gets large.

1.10. Theorem. Let $P$ be an integral polygon. There is a number $N_{p}$ such that if $N>N_{p}$, then $G_{N} P$ is composed of a connected component, together with a set of isolated vertices whose number depends only on $P$.

Indeed, the isolated vertices are associated to the corners of $P$ (as we shall see in $\S 3$ in the proof of 1.10 ).

Not every well-covered graph is $G_{1} P$ for some polygon $P$. The following is proved in $\S 4$. 


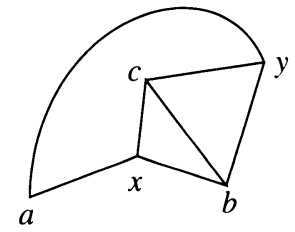

G

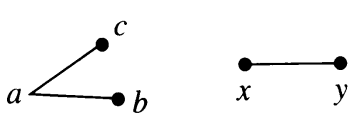

$K(G)$

FIGURE 3

1.11. Theorem. If $P$ is an integral polygon and $a(P)>1$, then $K_{1} P$ is a pl-disk of dimension $3 a(P)-\frac{1}{2} L(P)-1$.

Figure 3 shows that not every well-covered $G$ has $K(G)$ a disk.

Let $P$ be an integral polygon, and let $\operatorname{Aut}_{N} P$ be the group of $p \mathbf{Z}_{N}$ homeomorphisms of int $P$. By 1.3 , Aut $1 P$ surjects to $\mathrm{Z} / L(P)$, with kernel $\operatorname{Aut}_{1}(P, \partial)$ the elements fixing $P$. Now $\operatorname{Aut}_{1}(P, \partial)$ is clearly related to braid groups: as noted by Delaney in [D], an element of Aut $_{1} P$ permutes the elements of int $P \cap \frac{1}{N} \mathbf{Z}^{2}$, so we have homomorphisms from $\operatorname{Aut}_{1}(P, \partial)$ to the braid group on \#(int $\left.P \cap \frac{1}{N} \mathbf{Z}\right)$ strings.

We now show that $\operatorname{Aut}_{0} P$ acts on $K(P)$ for any integral polygon $P$ and that one can define the limit $K(P)=\lim _{\longrightarrow} K_{N} P$ of the $K_{N} P$. Thus, $K(P)$ is a sort of "complex of curves" [I] for the group Aut $_{0} P$.

1.12. Proposition. Let $P$ be an integral polygon. Then for all $n, N \in \mathbf{Z}$ there is a pl-embedding $i: K_{N} P \rightarrow K_{N n} P$. Further, for all $n, m, N \in \mathbf{Z}$ the following diagram commutes:

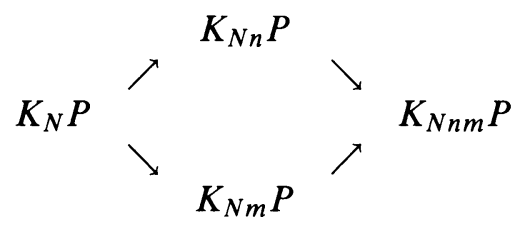

Proof. We first define $i$ on vertices. If $t=\overline{a b}$ is an $N$-segment, then $S_{0}(t)=$ $\overline{a a+(b-a) / n}, \ldots, S_{n-1}(t)=\overline{b-(b-a / m) b}$ are $N n$-segments. Define $i(t)$ to be the barycenter of the $n-1$ simplex $s(t)=\left(S_{0}(t), \ldots, S_{n-1}(t)\right)$. Now if $t=\left(t_{0}, \ldots, t_{k}\right)$ is a $k$-simplex in $K_{N}(P)$, then $i(t)$ is defined to be the convex closure of the $i\left(t_{j}\right)$ in the simplex $s\left(t_{0}\right) * \cdots * s\left(t_{k}\right)$. Naturality follows from the definition.

1.13. Definition. $K(P)$ is the direct limit of the $K_{N} P$, the limit taken over the natural numbers with maps $N \rightarrow n N$.

1.14. Proposition. The length function extends to a function $L: K(P) \rightarrow \mathbf{R}$.

Proof. First we define $L$ restricted to $K_{N} P$. On each vertex $t$ of $K_{N} P$, we have $L(t)=\frac{1}{N}$. Suppose that $L$ is defined on $(k-1)$-simplices of $K_{N} P$. If $t=\left(t_{0}, \ldots, t_{k}\right)$ is a $k$-simplex, then $L$ is defined on $\partial t$. Define $L$ to be $(k+1) / N$ on the barycenter of $t$, and extend to the rest of $t$ by "coning off".

It is evident that $L$ commutes with the $i_{N, n N}$ and is therefore defined on $K(P)$. 
1.15. Proposition. The group $\mathrm{Aut}_{0} P$ of $p \mathbf{Z}_{0}$ homeomorphisms of $\overline{\operatorname{int} P}$ acts continuously on $K(P)$, and $L$ is invariant under the action.

Proof. Let $g \in \operatorname{Aut}_{0} P$, and let $s=\left(s_{0}, \ldots, s_{k}\right)$ be a $k$-simplex in $K_{N} P$. If $g$ is linear on each $s_{i}$, define $g s$ to be the simplex $\left(g s_{0}, \ldots, g s_{k}\right)$. If $g$ is not linear on the $s_{i}$, there is some subdivision of the $s_{i}$ on which $g$ is linear, and can thus be defined.

\section{TRIANGUlations AND $p \mathbf{Z}$ HOMEOMORPHISMS}

We begin with a simple observation.

2.1. Lemma. Let $T_{1}$ and $T_{2}$ be 1-triangles, with vertices $a_{i} \in T_{i}$. There is a unique element $g \in A_{1}$ such that $g T_{1}=T_{2}$ and $g a_{1}=a_{2}$.

Proof. Composing with translations, we can assume that $a_{1}=a_{2}=(0,0)$. Recall from the proof of Lemma 1.6 that the remaining sides of each of the $T_{i}$ form a basis for $\mathbf{Z}^{2}$. The lemma follows.

Lemma 2.1 gives an interesting way to construct $p \mathbf{Z}$ homeomorphisms. Suppose that $P$ and $Q$ are integral polygons with 1-triangulations which are combinatorially the same. Then (see Figure 4) applying Lemma 2.1 to each pair of corresponding 1-triangles constructs a well-defined $p \mathbf{Z}$ homeomorphism from $\overline{\text { int } P}$ to int $Q$ (Figure 4) which we call a simple homeomorphism.

2.2. Definition. Let $P$ and $Q$ be integral polygons. Then $f: \overline{\operatorname{int} P} \rightarrow \overline{\operatorname{int} Q}$ is a 1-triangulated homeomorphism if

(i) $f$ is simple,

(ii) $f$ is a composite of 1-triangulated homeomorphisms or

(iii) int $P=$ int $P_{1} \cup$ int $P_{2}$, int $Q=\overline{\text { int } Q_{1}} \cup \overline{\text { int } Q_{2}}$, where $P_{i}$ and $Q_{i}$ are integral polygons, int $P_{1} \cap \overline{\text { int } P_{2}}=\overline{v_{0} \cdots v_{n}}$, int $Q_{1} \cap \overline{\text { int } Q_{2}}=\overline{w_{0} \cdots w_{n}}$, with $\overline{v_{i} v_{i+1}}$ and $\overline{w_{i} w_{i+1}}$ 1-segments, and $f_{i}: \overline{\text { int } P_{i}} \rightarrow \overline{\text { int } Q_{i}}, i=1,2$, are 1triangulated homeomorphisms such that $f_{i}\left(v_{j}\right)=w_{j}$. Then, defining $f: \overline{\operatorname{int} P}$ $\rightarrow \overline{\text { int } Q}$ by setting $f \mid p_{i} \equiv f_{i}, f$ is a 1-triangulated homeomorphism.

2.3. Remarks. (a) Condition (iii) could be replaced by defining "immersed polygons".

(b) 1-triangulated homeomorphisms are clearly $p \mathbf{Z}_{1}$ homeomorphisms, but the reverse is not true: let $P$ be the triangle with vertices $(0,0),(1,0)$, and $(0,1)$ (see Figure 5). The 1-triangulated homeomorphisms form a cyclic group of order 3. However, the homeomorphism pictured in the figure is $p \mathbf{Z}_{1}$ for all $n$.

The following is evidently stronger than Theorem 1.3.
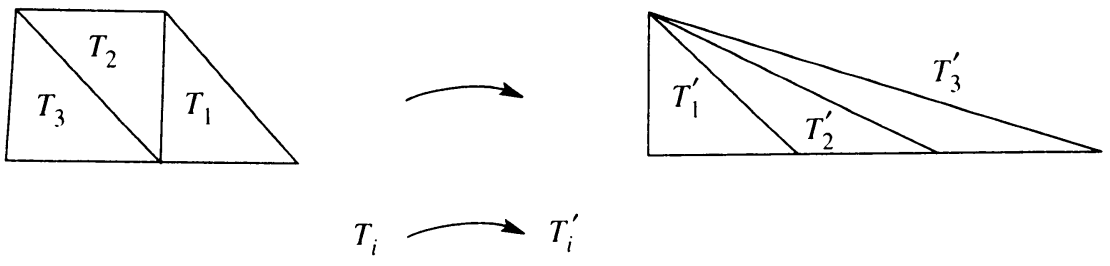

Figure 4 

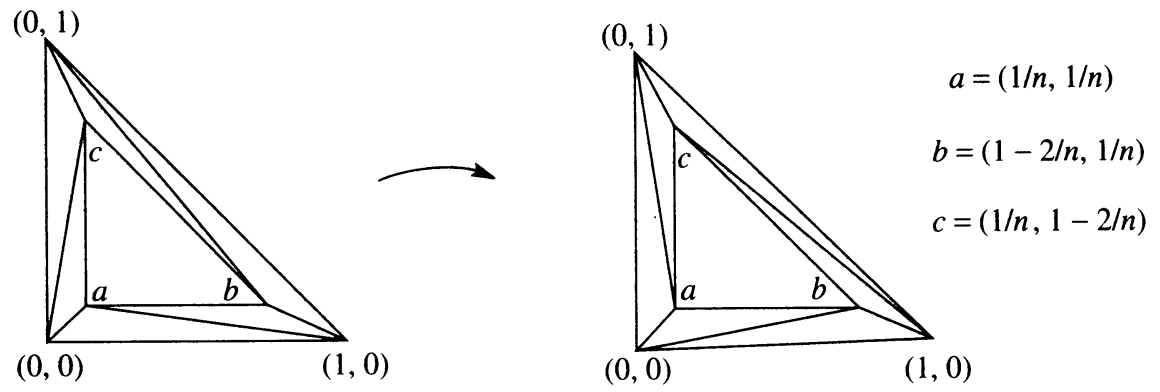

FIGURE 5

2.4. Theorem. Let $P$ and $Q$ be integral polygons of equal area and length, and let $p \in P \cap \mathbf{Z}^{2}$ and $q \in Q \cap \mathbf{Z}^{2}$. Then there exists a 1-triangulated homeomorphism $f: \overline{\operatorname{int} P} \rightarrow \overline{\operatorname{int} Q}$, with $f(p)=q$.

Conjecture. The group of $p \mathrm{Z}$ homeomorphisms of the interior of an integral polygon $P$ is the same as the group of 1-triangulated homeomorphisms. In particular, the group is finitely generated, and the group of $p \mathbf{Z}$ homeomorphisms of a 1-triangle is simply the group $\mathrm{Z} / 3$ of rotations.

Note that the group of $p \mathbf{Z}_{1}$ homeomorphisms of a 1-triangle is not finitely generated (see Figure 5).

Several preliminary notions are necessary for the proof. We shall write the integral points of $P$ and $Q$ in counterclockwise order as $p=p_{0}, \ldots, p_{L-1}$ and $q=q_{0}, \ldots, q_{L-1}$, where $L=L(P)=L(Q)$. If $a, b$, and $c$ are points on a polygon, then $a<b<c$ means that $c$ follows $b$, which follows $a$, in counterclockwise order. If $0 \leq i, j \leq L-1$, then we take $j-i$ to mean the element of $j-i+L Z$ between 0 and $L-1$.

If $S$ is an integral polygon with vertices $S \cap \mathbf{Z}^{2}=\left\{s_{0}, \ldots, s_{n}\right\}$, then a side triangle is a 1-triangle of the form $\overline{s_{i} S_{i+1} S_{i+2} S_{i}}$, and an inner triangle is a 1-triangle of the form $\overline{s_{i} S_{i+1} v s_{i}}$, where $v \in$ int $S \cap \mathbf{Z}^{2}$.

2.5. Lemma. In any 1-triangulation of an integral polygon, either a side triangle or an inner triangle must occur.

Proof. Let $S$ be an integral polygon with $S \cap \mathbf{Z}^{2}=\left\{s_{0}, \ldots, s_{n}\right\}$. Suppose there is no inner triangle in a given triangulation. Then each $\overline{s_{i} s_{i+1}}$ is the edge of a triangle $\overline{s_{i} s_{i+1} S_{f(i)} s_{i}}$ with $s_{i} \leq s_{i+1}<s_{f(i)}$. Let $j$ be an index minimizing $f(i)-i$. If $f(j) \neq j+2$, then $s_{j+1}<f(j+1) \leq f(j)$, whence $f(j+1)-(j+1)<$ $f(j)-j$, a contradiction.

2.6. Corollary. (a) If \#(int $\left.S \cap \mathbf{Z}^{2}\right)=0$, then any triangulation contains a side triangle.

(b) If $L(S)=3$, then any triangulation has an inner triangle.

Proof of 2.4. The proof is by induction on $2 a(P)$. When $a(P)=\frac{1}{2}, P$ and $Q$ are 1-triangles, and we apply Lemma 2.1 . In the general case, we will apply Lemma 2.5 to reduce the area of $P$ and $Q$.

Assume first that \#(int $\left.P \cap \mathbf{Z}^{2}\right)=0$. By Corollary 2.6, $P$ and $Q$ have side triangles $T_{P}=\overline{p_{i} p_{i+1} p_{i+2} p_{i}}$ and $T_{Q}=\overline{q_{j} q_{j+1} q_{j+2} q_{j}}$. Let $S$ be an integral polygon with $L(S)=L(P)$, \#(int $\left.S \cap \mathbf{Z}^{2}\right)=0, S \cap \mathbf{Z}^{2}=\left\{S_{0}, \ldots, S_{L-1}\right\}$, 


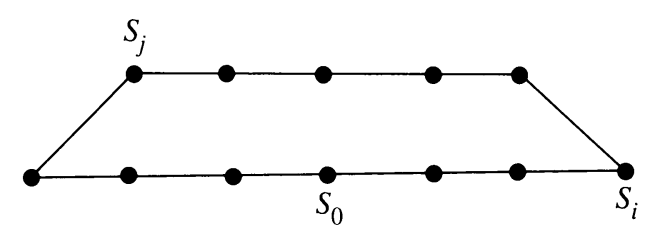

FIGURE 6

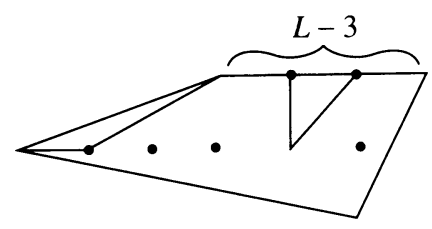

(a)

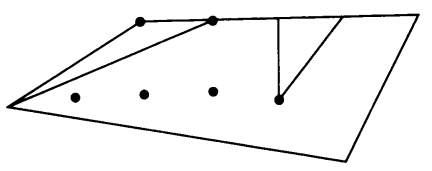

(b)

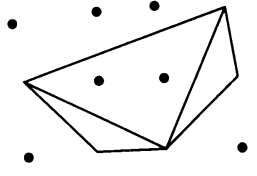

(c)

Figure 7. (a) Two inner triangles; (b) one inner, one side; (c) two side triangles (begin with the inner triangle, which contains all of int $S \cap \mathbf{Z}^{2}$, and then add side triangles).

which has side triangles $\overline{s_{i} S_{i+1} S_{i+2} s_{i}}$ and $\overline{s_{j} S_{j+1} S_{j+2} S_{j}}$ (Figure 6 indicates the construction of $S$ ).

Now use Definition 2.2(iii) and induction to construct 1-triangulated homeomorphisms $\overline{\operatorname{int} P} \rightarrow \overline{\operatorname{int} S}$ and $\overline{\operatorname{int} S} \rightarrow \overline{\operatorname{int} Q}$, which take $p$ to $S_{0}$, and $S_{0}$ to $q$, and we are done.

If \#(int $\left.P \cap \mathbf{Z}^{2}\right)>0$, we reason as above; the situation is more complicated because $P$ and $Q$ have either a side or inner triangle, and we must show that there exist integral polygons $S$ with $L(S)=L(P)$, \#(int $\left.S \cap \mathbf{Z}^{2}\right)=$ $\#$ (int $P \cap Z^{2}$ ), which admit both sorts of triangles in all possible positions (these $S$ are displayed in Figure 7). Repeating the argument above concludes the proof.

\section{LOCAL AND GLOBAL STRUCTURE OF $G(P)$}

Recall the graph $G_{1}(P)$ (see $\S 1$ ) whose vertices are 1-segments whose interiors lie in the interior of the integral polygon $P$, and with an edge between two vertices if the corresponding 1 -segments cross. Our goal in this section is to prove Theorem 1.10, which we paraphrase as follows: for each $P$ there is some $N_{P}$ such that, if $N>N_{P}, G_{1}(N P)$ consists of a connected graph with some isolated vertices whose number depends on $P$. Our approach is inspired by ideas from analysis. As it turns out, the isolated vertices in $G_{1}(N P), N>N_{p}$, are associated to the corners of $P$; we make a brief study of the graphs of sectors between two rays. Then, a family of "patches"-integral polygons with connected graphs-is produced. The proof of Theorem 1.10 involves these large and small scales.

We begin with the small-scale picture.

3.1. Definition. A patch is an integral polygon $P$ so that $G_{1}(P)$ is connected, and, if $\overline{a b}$ is any 1 -segment in $\mathbf{R}^{2}$ such that $\overline{a b} \cap$ int $P$ is nonempty, then some 1-segment in int $P$ crosses $\overline{a b}$. 
Let $R_{n, k}$ be the rectangle with corners $(0,0),(n, 0),(n, k),(0, k)$.

3.2. Proposition. For any $g \in A_{1}, g R_{n, k}$ is a patch.

Proof. Since elements of $A_{1}$ preserve graphs, it suffices to show that $R_{n, k}$ is a patch. If $\overline{a b}$ is a 1 -segment and $\overline{a b} \cap$ int $R_{n, k} \neq \varnothing$, then there is some square $S=\overline{(x, y)(x+1, y)(x+1, y+1),(x, y+1)}$ whose interior is contained in int $R_{n, k}$, such that $\overline{a b} \cap$ int $S \neq \varnothing$. But then one of the diagonals of $S$ crosses $\overline{a b}$.

3.3. Corollary (of the proof). If $P$ is an integral polygon such that $\overline{\operatorname{int} P}$ is the union of interiors of squares, then $P$ is a patch.

Such a $P$ is called a block polygon.

3.4. Proposition. The union of two overlapping patches is a patch: Let $P, P_{1}$, and $P_{2}$ be integral polygons, let $P_{1}$ and $P_{2}$ be patches, and let $\overline{\operatorname{int} P}=\overline{\operatorname{int} P_{1}} \cup$ int $P_{2}$. Then $P$ is a patch.

Proof. Since $P_{1}$ and $P_{2}$ overlap, there is some 1-segment whose interior is contained in int $P_{1} \cap$ int $P_{2}$. Thus $G_{1}(P)$ is connected. If $\overline{a b}$ is a 1 -segment which has nonempty intersection with int $P$, then it has nonempty intersection with int $P_{1}$ or int $P_{2}$. Thus $P$ is a patch.

Let us now discuss graphs associated to noncompact regions. If $R$ is the closure of an open region in $\mathbf{R}^{2}$ whose boundary is the union of 1-segments, then we denote by $G_{1}(R)$ the graph whose vertices are 1-segments whose interiors are contained in int $R$, with an edge between two vertices if the corresponding 1 -segments cross.

Consider first a half-plane, that is, $R=\{(x, y): a x+b y \geq c, a, b, c \in \mathbf{Q}\}$. Applying an element of $A_{1}$, we can assume that $R=\{(x, y): y \geq 0\}$. Then $R=\bigcup \overline{\operatorname{int} P_{n}}$, where $P_{n}$ is the rectangle with corners $( \pm n, 0),( \pm n, n)$. Since each $P_{n}$ is a patch, $G_{1}(R)$ is connected, thus:

\subsection{Proposition. $R$ is a patch.}

That is to say, if $\overline{a b}$ is a 1 -segment of $\mathbf{R}^{2}$ whose interior has nonempty intersection with the interior of $R$, then some 1-segment in the interior of $R$ crosses $\overline{a b}$.

If $v=(a, b)$ and $w=(c, d)$, with $a, b$, and $c, d$ relatively prime, let $r$ and $s$ be the rays from $(0,0)$ through $v$ and $w$ respectively. Then the angle $A(w, v)$ is the region swept out by a ray sweeping counterclockwise from $s$ to $r$. If $A(w, v)$ (properly) contains a half-plane it is called (strictly) concave, and if not, convex.

Every concave angle $A(w, v)$ is the union of two overlapping half-planes. Applying Propositions 3.4 and 3.5, we find

\subsection{Proposition. If $A(w, v)$ is concave, it is a patch.}

The image of a concave, strictly concave, or convex angle under an element of $A_{1}$ which takes $(0,0)$ to a point $p$ will also be called a concave, strictly concave, or convex angle at $p$.

3.7. Definition. Let $A(w, v)$ be a strictly concave angle, and let $M \in \mathbf{Z}, M>$ 0 . The $M$-cap for $A(w, v)$ is the polygon $P=P_{M}(w, v)$ so that $\overline{\operatorname{int} P}=$ 
$\overline{\text { int } R_{1}} \cup \overline{\text { int } R_{2}}$, where

$$
R_{1}=\overline{M v,-M v,-M v-M w, M v-M w, M v}
$$

and

$$
R_{2}=\overline{M w, M w-M v,-M w-M v-M w, M w}
$$

(see Figure 8). An $M$-cap for an angle at $p$ is the image under some element of $A_{1}$ of an $M$-cap at $(0,0)$.

Note that since $v=(a, b)$ and $w=(c, d)$, with $a, b$ and $c, d$ relatively prime, $\overline{(0,0) v}$ and $\overline{(0,0) w}$ are 1 -segments. Hence, for example, $L\left(P_{M}(w, v)\right)=8 M$.

The situation for convex angles is more interesting. We will see that $G_{1}(A(w, v))$ consists of a connected piece and a number of isolated vertices.

If $A(w, v)$ is the image under $g \in \mathrm{SL}_{2} \mathrm{Z}$ of $A((1,0),(0,1))$, then we call $A(w, v)$ a right angle.

\subsection{Lemma. Right angles are patches.}

Proof. It suffices to prove $A((1,0),(0,1))$ is a patch. But $A((1,0),(0,1))$ is the union of $\overline{\operatorname{int} R_{n, n}}$, where $R_{n, n}$ is the square with corners $(0,0),(n, 0)$, $(0, n)$, and $(n, n)$, and $R_{n, n}$ is a patch by 3.2 .

3.9. Definition. Let $A(w, v)$ be convex. A chain from $w$ to $v$ is a sequence $w=v_{0}, v_{1}, \ldots, v_{n}, v_{n+1}=v$ with $v_{i}=\left(a_{i}, b_{i}\right)$, such that the rays from $(0,0)$ to $v_{i}$ are in $A(w, v)$ and occur in counterclockwise order, and such that

$$
\operatorname{det}\left(\begin{array}{cc}
a_{i} & b_{i} \\
a_{i+1} & b_{i+1}
\end{array}\right)=1, \quad 0 \leq i \leq n,
$$

that is, each $A\left(v_{i}, v_{i+1}\right)$ is a right angle.

3.10. Lemma. If $A(w, v)$ is convex, then there exists a chain from $w$ to $v$.

Proof. Applying an element of $\mathrm{SL}_{2} \mathbf{Z}$, we can assume that $v=(0,1)$ and $w=(a, b)$, with $b / a<1$. Considering Farey series [R] we can write

$$
\frac{b}{a}=\frac{p_{l}+p_{r}}{q_{l}+q_{r}}
$$

where $0 \leq p_{l} / q_{l}<b / a<p_{r} / q_{r} \leq 1$, and $b q_{r}-p_{r} a=-1$. Taking $w=$ $v_{0}, v_{1}=\left(q_{r}, p_{r}\right)$, and iterating, we will eventually arrive at $v_{n}=(1,1)$. Setting $v_{n+1}=v=(0,1)$ we have a chain.

As an example, take $v=(0,1)$ and $w=(5,3)$. Then $\frac{3}{5}=\frac{1+2}{2+3}$ and $\frac{2}{3}=\frac{1+1}{2+1}$, so the chain is $w=(5,3), v_{1}=(3,2), v_{2}=(1,1), v=(0,1)$.

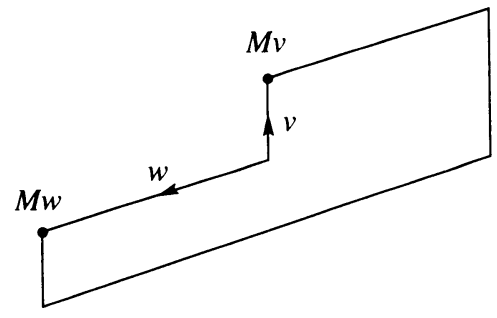

$M=2$

FIGURE 8 


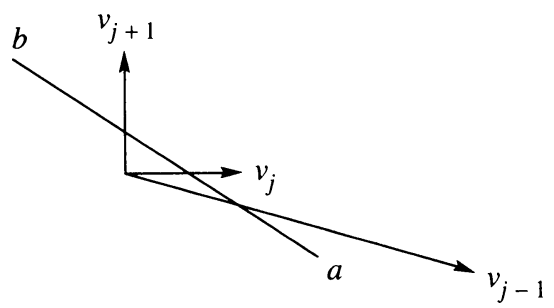

Figure 9

3.11. Proposition. Let $A(w, v)$ be a convex angle and $w=v_{0}, \ldots, v_{n+1}=v$ be a chain. There exist $N_{i}$ such that the isolated vertices of $G_{1}(A(w, v))$ are 1 -segments $\overline{k v_{i},(k+1) v_{i}}, 0 \leq k \leq N_{i}-1$. The complement of the collection of these vertices is a connected subgraph of $G_{1}(A(w, v))$.

Proof. By 3.8, each $A\left(v_{i}, v_{i+1}\right)$ is a patch. Further, it is clear that for each $i$, there is some $M_{i}$ so that $\overline{m v_{i},(m+1) v_{i}}$ is connected to $G_{1}\left(A\left(v_{i}, v_{i+1}\right)\right)$ and $G_{1}\left(A\left(v_{i-1}, v_{i}\right)\right)$ for $m \geq M_{i}$. Let $N_{i}$ be the smallest such $M_{i}$. Then the subgraph of $G_{1}(A(w, v))$ whose vertices are all but the $\overline{m v_{i},(m+1) v_{i}}, m<$ $N_{i}$, is connected. We must show that the vertices $\overline{m v_{i},(m+1) v_{i}}, m \leq N_{i}-1$, are indeed isolated. But if $\overline{a b}$ crosses $\overline{m v_{i},(m+1) v_{i}}$, then $\overline{a+v_{i}, b+v_{i}}$ crosses $\overline{(m+1) v_{i},(m+2) v_{i}}$, and so on, contradicting the definition of $M_{i}$.

The $N_{i}$ in 3.11 is called the weight of the singular vector $v_{i}$, if $N_{i} \leq 1$. The number of isolated vertices in $G_{1}(A(w, v))$ is $\sum N_{i}$.

Partially order the set of chains from $w$ to $v$ by inclusion.

3.12. Theorem. Given a convex angle $A(w, v)$, there is a minimal chain $w=$ $v_{0}, \ldots, v_{n+1}=v$. Each $v_{i}$ has positive weight.

Proof. Let $w=v_{0}, \ldots, v_{n+1}=v$ be a chain. We show that if a $v_{j}$ has weight 0 , then we can replace the chain with a subchain of cardinality strictly less.

If $\overline{0 v}_{j}$ is not isolated in $G_{1}(A(w, v))$, then some 1-segment $\overline{a b}$ crosses $\overline{0 v}_{j}$. Apply an element of $\mathrm{SL}_{2} \mathbf{Z}$ so that $v_{j}=(1,0), v_{j+1}=(0,1)$, and $v_{j-1}=(n,-1)$ for some $n \in \mathbf{N}$ (see Figure 9), and take $a$ with $y$ coordinate negative, and $b$ with $y$ coordinate positive. The 1 -segment $\overline{a b}$ crosses some number $m$ of $\overline{0 v}_{i}$. We prove, by induction on $m$, that the size of the chain can be reduced.

If $m=1$, then $\overline{a b}$ crosses only $\overline{0 v}_{j}$. From Figure 9 one sees that $\overline{a b}=$ $\overline{v_{j-1} v_{j+1}}$, in which case $v_{j-1}=(1,-1)$, so that $A\left(v_{j-1}, v_{j+1}\right)$ is a right angle, and $v_{j}$ can be dropped from the chain.

Assume that $m, n>1$. Then $\overline{a b}$ crosses $\overline{0 v}_{j-1}$, and it either crosses $\overline{0 v}_{j+1}$ or not. If $\overline{a b}$ does not cross $\overline{0 v}_{j+1}$, then $\overline{a v}_{j}$ crosses $\overline{0 v}_{j-1}$; by replacing $\overline{a b}$ with $\overline{a v}_{j}$ we can reduce $m$ and by induction we can reduce the length of the chain. If $\overline{a b}$ crosses both $\overline{0 v}_{j-1}$ and $\overline{0 v}_{j+1}$, then either $\overline{a v}_{j}$ crosses $\overline{0 v}_{j-1}$, or $\overline{b v}_{j}$ crosses $\overline{0 v}_{j+1}$. Either way $m$ is reduced, and by induction the chain is reduced.

To prove 1.10, we need a finite version of 3.12. If $A(w, v)$ is a right angle, the $M$-square $S_{M}(w, v)$ at $A(w, v)$ is the parallelogram with corners $(0,0)$, $M w, M v, M(w+v)$. If $A(w, v)$ is convex, let $w=v_{0}, \ldots, v_{n+1}=v$ be 


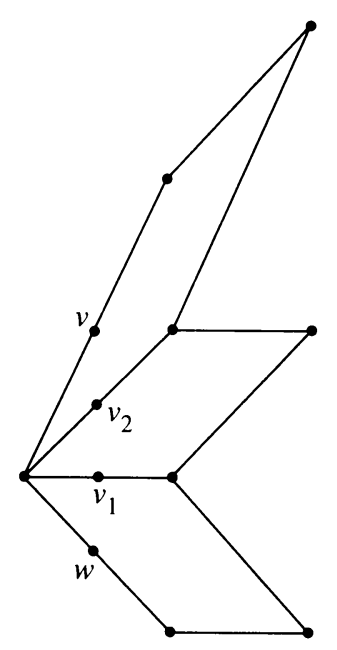

FIGURE 10. 2-pencil point

the minimal chain. The polygon $P_{M}(w, v)$ so that $\overline{\operatorname{int} P_{M}}=\bigcup \overline{\operatorname{int} S_{M}\left(v_{i}, v_{i+1}\right)}$ is called the $M$-pencil point at $A(w, v)$ (see Figure 10).

3.13. Lemma. Let $A(w, v)$ be a convex angle, and let $w=v_{0}, \ldots, v_{n+1}=v$ be a minimal chain with weights $N_{i}$. If $M>\max N_{i}$, then $G_{1}\left(P_{M}(w, v)\right)$ consists of a connected component with $\sum N_{i}$ isolated vertices $\overline{k v_{i},(k+1) v_{i}}$, $0 \leq k<N_{i}, 1 \leq i \leq n$.

Proof. The $M$-squares $S_{M}\left(v_{i}, v_{i+1}\right)$ are patches, so it suffices to show that the vertices $\overline{k v_{i},(k+1) v_{i}}, k \geq N_{i}$, are connected to the $G_{1}\left(S_{M}\left(v_{i}, v_{i+1}\right)\right)$ and $G_{1}\left(S_{M}\left(v_{i-1}, v_{i}\right)\right)$. With an element of $\mathrm{SL}_{2} \mathrm{Z}$, we can take $v_{i}=(1,0)$, $v_{i+1}=(0,1)$, and $v_{i-1}=(2 m+\varepsilon,-1)$ with $\varepsilon=0$ or 1 . It is not hard to check that $v_{i}$ has weight $N_{i}=m-1$, and that $\overline{(n ; 0)(m+1,0)}$ is crossed by $(2 n+\varepsilon,-1)(1-\varepsilon, 1)$.

Proof of Theorem 1.10. We prove that if $P$ is an integral polygon, then there is some $N_{p}$ such that, if $N>N_{p}, G_{1}(N P)$ consists of a connected component and $m_{p}$ isolated vertices. Here $m_{p}=\sum_{j} \sum_{i} N_{i}$, the sum over the weights of the singular vectors associated to minimal chains of each convex angle in $P$.

Begin by taking $N$ large enough so that $M$-caps or $M$-pencil points can be placed at each angle in $P$, where $M$ is larger than $\max M_{i}$ (Figure 11(a)).

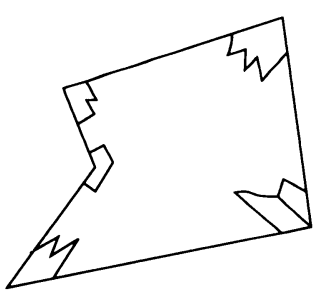

(a)

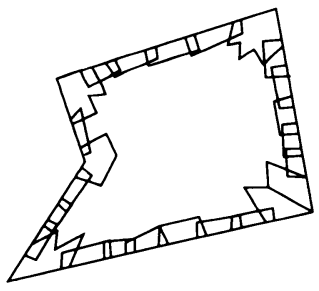

(b)

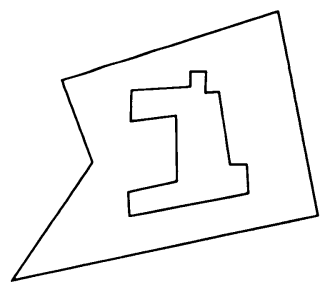

(c)

FigURE 11 
Now (Figure 11(b)) enlarging $N$ if necessary, translate the outer $M$-squares or rectangles of the pencil points and caps along their respective sides, so that each point in $P \cap Z^{2}$ is contained in one of the translated squares or rectangles. Finally, enlarge $N$ to an $N_{p}$ so that (possibly increasing $M$ ) there is a block polygon (recall 3.3) which overlaps the union of the squares and rectangles (Figure 11(c)). Applying 3.4, we are done.

\section{THE COMPLEX $K_{1} P$}

The object of this section is to prove that if $P$ is an integral polygon whose area is at least $\frac{3}{2}$, then $K_{1} P$ is a combinatorial disk. We know from 1.9 that $K_{1} P$ is a pure simplicial complex (that is, all maximal simplices have the same dimension) of dimension

$$
\operatorname{dim} K_{1} P=2 a(P)+N(P)-2,
$$

where $N(P)=\#\left(\right.$ int $\left.P \cap Z^{2}\right)$. Also, from $\S 3, G_{1} P$ often has isolated vertices, so that $K_{1} P$ is a cone. With some simple examples, these remarks lead to the suspicion that $K_{1} P$ is a (piecewise-linear) disk.

The proof that $K_{1} P$ is a disk is by induction and requires a generalization of the idea of polygon, which we approach as follows. If $K_{1} P$ is a disk, it is first of all a manifold, so that the link $\operatorname{Lk}(s)$ of each simplex $s$ should be a disk or a sphere. These $\operatorname{Lk}(s)$ can be seen as $K_{1} P_{s}$, where $P_{s}$ is a generalized polygon, called a "slit polygon."

Suppose $s=\left(s_{0}, \ldots, s_{k}\right)$, where the $s_{i}$ are 1 -segments in int $P$. Then an $m$ simplex $t=\left(t_{0}, \ldots, t_{m}\right)$ is in $\operatorname{Lk}(s)$ if and only if $s * t=\left(s_{0}, \ldots, s_{k}, t_{0}, \ldots\right.$, $\left.t_{m}\right)$ is a simplex in $K_{1} P$; in other words, none of the $t_{i}$ cross any $s_{j}$. We think then of $t$ as a simplex in $K_{1} P_{s}$, where $P_{s}$ is the polygon $P$, slit at each $s_{i}$.

By $a\left(P_{s}\right)$ we mean $a(P) ; N\left(P_{s}\right)$ is $N(P)$ less the number of points in int $P \cap \mathbf{Z}^{2}$ which are endpoints of some $s_{i}$. Then equation (4.1) holds for slit polygons. By int $P_{s}$ we mean int $P-\bigcup S_{i}$, and $h\left(P_{s}\right)=\operatorname{rank} H_{1}\left(\right.$ int $\left.P_{s}\right)$. If $t$ is a simplex in $K_{1}\left(P_{s}\right)=\mathrm{Lk}(s)$, we define $\left(P_{s}\right)_{t}=P_{s * t}$, so we can "slit" slit polygons. The number of components of $P_{s}$ means the number of components of int $P_{s}$; each component of int $P_{s}$ is int $Q$ for some slit polygon $Q$, and we speak of the components $Q_{1}, \ldots, Q_{n}$ of $P_{s}$. The following remark is important for the sequel.

4.2. Lemma. If the components of $P_{s}$ are $Q_{1}, \ldots, Q_{n}$, then $K_{1}\left(P_{s}\right)=K_{1}\left(Q_{1}\right) *$ $K_{1}\left(Q_{2}\right) * \cdots * K_{1}\left(Q_{n}\right)$.

Let $P$ be an integral polygon, $s$ a simplex in $K_{1} P$, and consider the slit polygon $P_{s}$. Then int $P_{s}$ is the interior of a pl-manifold with boundary which submerges onto int $\bar{P}$ (see Figure 12). We will call this closed manifold $\overline{\operatorname{int} P_{s}}$. By $\partial P_{s}$ is meant the component of the boundary of $\overline{\operatorname{int} P_{s}}$ whose image in $\overline{\operatorname{int} P}$ contains $P=\partial \overline{\operatorname{int} P}$. Note that $\partial P_{s}$ can be described as a series $\overline{P_{1} P_{2} \cdots P_{n} P_{1}}$ of points in $\mathbf{Z}^{2}$ such that each $p_{i} p_{i+1}$ is a 1-segment in counterclockwise order (Figure 12). By an angle of $P_{s}$ is meant a 3-point fragment $\overline{p_{i} p_{i+1} p_{i+2}}$ of $\partial P_{s}$.

We will prove the following version of 1.11 .

4.3. Theorem. If $P$ is a connected slit polygon and $a(P)>1$ or $N(P) \leq 1$, then $K_{1}(p)$ is a pl-disk. 


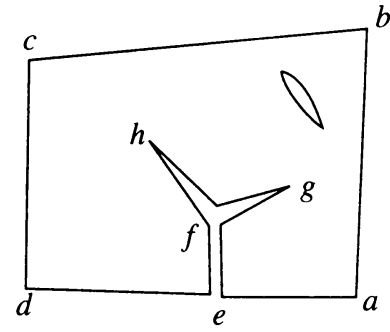

$\overline{\text { int } P_{s}}$

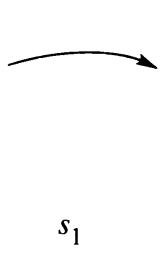

1

$$
\begin{gathered}
\partial P_{s}=\overline{\text { abcdefhfgfea }} \\
s=\overline{\left(s_{0}, s_{1}, s_{2}, s_{3}\right)}
\end{gathered}
$$

FIGURE 12

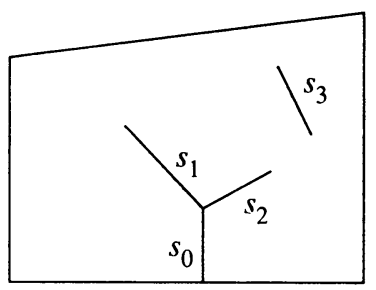

$\overline{\text { int } P}$

To begin, consider the connected slit polygons with area $\frac{3}{2}$ or less. If $a(P)=$ $\frac{1}{2}$, then $P$ is a 1 -triangle and $K_{1}(P)$ is empty. If $a(P)=1$, then $P$ is two 1 -triangles joined at a face, so $K_{1}(P)$ is a 0 -sphere $S^{0}$ or a point, that is, a 0-disk.

4.4. Lemma. If $P$ is a connected slit polygon and $a(P)=\frac{3}{2}$, then $K_{1}(P)$ is $a$ 1-disk or a 2-disk.

Proof. Suppose first that $P$ is not slit. Then, by Pick's theorem, either $N(P)=$ $1, L(P)=3$ or $N(P)=0, L(P)=5$. In the former case, there is a 1segment from the interior vertex to each of the three vertices of $P$, so $K_{1}(P)$ is a 2-simplex.

Suppose that $N(P)=0$ and $L(P)=5$. Label the points of $P \cap Z^{2}$ in counterclockwise order $a, b, c, d, e$. By 2.6, $P$ has a side triangle; without loss of generality we can assume that $\overline{a c}$ is a 1-segment in $\overline{\operatorname{int} P}$ and that $\overline{a b c a}$ is a 1-triangle. Composing with an element of $A_{1}$ we can assume that $a=(0,1), b=(0,0)$, and $c=(1,0)$.

If neither $\overline{b d}$ nor $\overline{b e}$ are 1 -segments in int $\bar{P}$, then $K_{1}(P)$ is the cone at the vertex $\overline{a c}$ of $K_{1}(\overline{a c d e})$, which is an $S^{0}$ or a $D^{0}$, and thus $K_{1}(P)$ is a 1-disk.

If at least one of $\overline{b d}$ or $\overline{b e}$ is a 1 -segment in $\overline{\operatorname{int} P}$, then one of $d$ or $e$ must be $(1,1)$ (Figure 13); without loss of generality, put $e=(1,1)$, whence $d=(2, k)$ for some $k \in \mathbf{Z}$ (Figure 13(a)). Then Figure 13(b), (c), (d) show that $K_{1}(P)$ is a 1 -disk.

Now, if $P$ is a connected slit polygon with area $\frac{3}{2}$, it must be $Q_{s}$, where $L(Q)=3$ and $s$ is a 1-segment from a point of $Q \cap \mathbb{Z}^{2}$ to the interior vertex. Thus $K_{1}(P)=K_{1}\left(Q_{s}\right)$ is a 1 -simplex.

Proof of 4.3. Put the triple $(a(P), N(P), h(P))$ in lexicographic order (e.g., $(2,1,1)>(1,4,6)>(1,3,8))$; the proof is by induction on the triples. Lemma 4.4 deals with the initial case $\left(\frac{3}{2}, 0,0\right)$. Let $\overline{a b c}$ be a convex angle in $\partial P$ (e.g., in Figure 12, $g f e$ ). Applying an element of $A_{1}$, we can put $b$ at $(0,0), a$ at $(0,1)$, and $c$ at $(n,-m), n, m \geq 0, m<n$. 


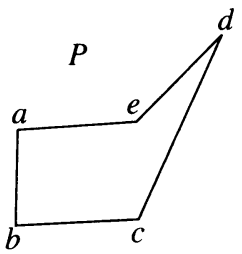

(a)

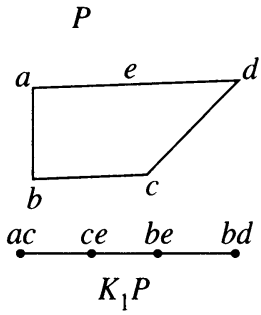

(b) $k=1$

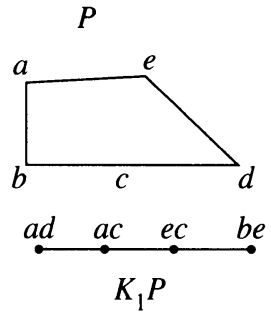

(c) $k=0$

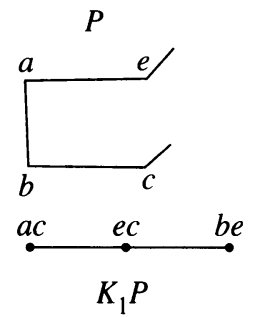

(d) $k \neq 0,1$

Figure 13

Suppose that $m>0$. Then (as in 3.12) the edge $s=\overline{(0,0)(1,0)}$ is an isolated vertex in $G_{1} P$, and so $K_{1} P$ is the cone at $S$ on $K_{1} P_{s}$. Now $a\left(P_{s}\right)=$ $a(P)$, but either $h\left(P_{s}\right)<h(P)$ or $N\left(P_{s}\right)<N(P)$, so, by induction, $K_{1} P_{s}$ is a disk and hence $K_{1} P$ is a disk.

Now suppose that $m=0$, so that $c$ is at $(1,0)$. Let $s=\overline{(0,1)(1,0)}$. If no 1 -segments of $\overline{\operatorname{int} P}$ have an endpoint at $(0,0)$, then $s$ is an isolated vertex in $G_{1} P$, and so $K_{1} P$ is the cone at $s$ on $K_{1} Q$, where $Q$ is $P$ with $\overline{a b c}$ replaced by $\overline{a c}$, that is, with the triangle $\overline{a b c a}$ excised. (Since $\overline{b c} \subset P, Q$ is still a slit polygon.) Since $a(Q)<a(P)$, by induction $K_{1} P$ is a disk.

If, on the other hand, some edge crosses $s$, then $(1,1) \in \overline{\operatorname{int} P}$ and $t=$ $\overline{(0,0)(1,1)}$ must be a 1 -segment in $P$. I claim any triangulation of $P$ must contain either $s$ or $t$ as an edge. For suppose some triangulation does not include $t$. Then some 1-segment $x$ of the triangulation crosses $t$. But any edge crossing $s$ would also cross $x$, and consequently $s$ is an edge in the triangulation.

Since either $s$ or $t$ is in any triangulation of $P$, it follows that $K_{1} P$ is the union of two cones: the cone at $s$ of $K_{1} P_{s}$ and the cone at $t$ of $K_{1} P_{t}$. The two cones intersect in $X=K_{1} P_{s} \cap K_{1} P_{t}$. If we can show that $X$ is a disk we are done (by, for example, Corollary II.16 of [GL]).

$X$ is the subcomplex of $K_{1} P$ whose simplices are partial triangulations of $P$ which contain no edges crossing $s$ or $t$. Let $s_{1}=\overline{(0,1)(1,1)}$ and $s_{2}=$ $\overline{(1,0)(1,1)}$. Either $s_{1}$ or $s_{2}$ or both are vertices in $K_{1} P$. If only one is a vertex in $K_{1} P$, say $s_{1}$, then $X$ is the cone at $s_{1}$ on $K_{1} Q$, where $Q$ is the slit polygon obtained by replacing $\overline{a b c}$ with $\overline{a(1,1) c}$ in $\partial P$. Since $a(Q)<a(P)$, by induction $K_{1} P$ is disk.

If both $s_{1}$ and $s_{2}$ are in $K_{1} P$, then $K_{1} P$ is the join of $K_{1} Q$ with the 1 -simplex $\left(s_{1}, s_{2}\right)$, and again is a disk.

\section{BIBLIOGRAPHY}

[D] R. L. Devaney, A piecewise linear model for the zones of instability of an area preserving map, Phys. D 10 (1984), 387-393.

[GL] L. C. Glaser, Geometrical combinatorial topology, Vol. I, Van Nostrand Reinhold, New York, 1970.

[G] M. C. Golumbic, Special issue on interval graphs and related topics, Discrete Math. 55 (1985), 113-123. 
[HT] A. Hatcher and W. Thurston, A presentation for the mapping class group of an oriented surface, Topology 19 (1980), 221-237.

[I] N. V. Ivanov, Complexes of curves and Teichmüller modular groups, Uspekhi Mat. Nauk 42 (1987), 49-91.

[R] H. Rademacher, Topics in analytic number theory, Springer, Berlin, 1973.

[GS] P. Greenberg and V. Sergiescu, An acyclic extension of the braid group, preprint.

[Gr] P. Greenberg, A combinatorial method for pseudogroups, preprint.

[GKW] R. W. Gaskell, M. S. Klamkin, and P. Watson, Triangulations and Pick's theorem, Math. Mag. 49 (1976), 35-37.

Département de Mathématiques, Université Louis Pasteur, 7 rue de Rene Descartes, 67084 Strasbourg Cedex, France

Current address: Institut Fourier, B.P. 74, Université de Grenoble, 38402 St. Martin d'Hères, France

E-mail address: greenber@frgren81.bitnet 